\title{
Population structure of drug-resistant Mycobacterium tuberculosis in Central Asia
}

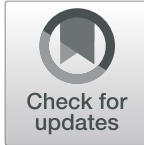

\author{
Anna Engström ${ }^{1,2,3,4,5^{*}}$ (D) Uladzimir Antonenka ${ }^{6}$, Abdylat Kadyrov ${ }^{7}$, Gulmira Kalmambetova ${ }^{8}$, Katharina Kranzer ${ }^{9}$, \\ Matthias Merker ${ }^{1}$, Olim Kabirov ${ }^{10}$, Nargiza Parpieva ${ }^{11}$, Asliddin Rajabov ${ }^{12}$, Evgeni Sahalchyk ${ }^{6}$, \\ Zayniddin Sayfudtinov ${ }^{13}$, Stefan Niemann ${ }^{1 \dagger}$ and Harald Hoffmann ${ }^{6,14,15+}$
}

\begin{abstract}
Background: Drug-resistant tuberculosis (TB) is a major public health concern threathing the success of TB control efforts, and this is particularily problematic in Central Asia. Here, we present the first analysis of the population structure of Mycobacterium tuberculosis complex isolates in the Central Asian republics Uzbekistan, Tajikistan, and Kyrgyzstan.

Methods: The study set consisted of 607 isolates with 235 from Uzbekistan, 206 from Tajikistan, and 166 from Kyrgyzstan. 24-loci MIRU-VNTR (Mycobacterial Interspersed Repetitive Units - Variable Number of Tandem Repeats) typing and spoligotyping were combined for genotyping. In addition, phenotypic drug suceptibility was performed.

Results: The population structure mainly comprises strains of the Beijing lineage (411/607). 349 of the 411 Beijing isolates formed clusters, compared to only 33 of the 196 isolates from other clades. Beijing 94-32 $(n=145)$ and 100-32 $(n=70)$ formed the largest clusters. Beijing isolates were more frequently multidrug-resistant, pre-extensively resistant (pre-XDR)- or XDR-TB than other genotypes.

Conclusions: Beijing clusters 94-32 and 100-32 are the dominant MTB genotypes in Central Asia. The relative size of 100-32 compared to previous studies in Kazakhstan and its unequal geographic distribution support the hypothesis of its more recent emergence in Central Asia. The data also demonstrate that clonal spread of resistant TB strains, particularly of the Beijing lineage, is a root of the so far uncontroled MDR-TB epidemic in Central Asia.
\end{abstract}

Keywords: Molecular typing, MIRU, MDR-TB, Beijing, Cluster

\section{Background}

Drug-resistant tuberculosis (TB) is a public health concern threathing the success of TB control efforts. This is particularily problematic in the Central Asian Republics (CARs) Uzbekistan, Tajikistan, and Kyrgyzstan where multidrug-resistant (MDR)-TB rates reach 24\%, 22\%, $27 \%$, and $63 \%, 45 \%$ and $60 \%$ among new and previously treated cases, respectively [1].

Little is known about the population structure of drug resistant Myocbacterium tuberculosis (MTB) strains in

\footnotetext{
*Correspondence: anna.engstrom@scilifelab.se; annaengstroem@gmail.com; anna.lyander@gmail.com

'Stefan Niemann and Harald Hoffmann contributed equally to this work. ${ }^{1}$ Molecular and Experimental Mycobacteriology, Research Center Borstel, Parkallee 1, 23845 Borstel, Germany

${ }^{2}$ Department of Medical Biochemistry and Microbiology, Uppsala University, IMBIM, Box 582, 75123 Uppsala, Uppsala, Sweden

Full list of author information is available at the end of the article
}

Central Asia. Available reports are limited by either outdated genotyping techniques, sampling of special populations and/or small sample sizes [2-4]. Here, we provide a snapshot of the population structure of MTB strains based on World Health Organization resistance surveys performed in Kyrgyzstan, Tajikistan, and Uzbekistan representing two thirds of the Central Asian population.

For genotyping we combined 24-loci MIRU-VNTR typing (Mycobacterial Interspersed Repetitive Units - Variable Number of Tandem Repeats) with spoligotyping. MIRU-VNTR provides high-resolution discrimination of strains for epidemiological studies and phylogenetic classification [5]. MIRU-VNTR data allow also to generate parsimonious phylogenetic networks such as minimumspanning trees (MST) [6]. These results were combined with phenotypic drug susceptibility data to determine the presence of highly resistant dominant clones.

(c) The Author(s). 2019 Open Access This article is distributed under the terms of the Creative Commons Attribution 4.0 International License (http://creativecommons.org/licenses/by/4.0/), which permits unrestricted use, distribution, and reproduction in any medium, provided you give appropriate credit to the original author(s) and the source, provide a link to the Creative Commons license, and indicate if changes were made. The Creative Commons Public Domain Dedication waiver (http://creativecommons.org/publicdomain/zero/1.0/) applies to the data made available in this article, unless otherwise stated. 


\section{Methods}

Seven hundred and seven clinical MTB isolates (306 from Uzbekistan, 216 from Tajikistan, and 185 from Kyrgyzstan) were collected in the framework of crosssectional national drug resistance surveys (DRS) [7, 8] and included in the study. The study population was biased towards resistant strains since only isolates that required retesting for quality control purposes as per the WHO DRS protocol which was valid at the time were included [8]. This approach artificially increased the proportion of MDR-,pre-extensively drug-resistant (pre-XDR) and
XDR-TB strains. Ethical approvals for the DRSs were granted by the respective national ethical committees.

DNA was extracted by heat lysis of bacteria from solid culture on LJ medium. 24-loci MIRU-VNTR genotyping and spoligotyping was performed as previously described $[5,9]$. Genotypes were identified on the MIRUVNTRplus web database (www.miruvntrplus.org) [5]. Spoligotyping data were used to confirm strain relationships and for genotype classification. Typing data was analysed with Bionumerics (v7.5; Applied Maths, SintMartens-Latem, Belgium). MST analysis based on

Table 1 Demographic data, genotypes, haplotypes and phenotypes of the Central Asian M. tuberculosis complex study population

\begin{tabular}{|c|c|c|c|c|c|c|c|c|c|c|c|c|}
\hline & \multicolumn{3}{|c|}{ All countries } & \multicolumn{3}{|l|}{ Uzbekistan } & \multicolumn{3}{|l|}{ Tajikistan } & \multicolumn{3}{|l|}{ Kyrgystan } \\
\hline & No. strains & $\%$ & SD & No. strains & $\%$ & SD & No. strains & $\%$ & SD & No. strains & $\%$ & $\mathrm{SD}$ \\
\hline Samples & 607 & & & 235 & 38.7 & & 206 & 33.9 & & 166 & 27.3 & \\
\hline \multicolumn{13}{|l|}{ Gender } \\
\hline Male & & 359 & 59.1 & 139 & 59.1 & & 120 & 58.3 & & 100 & 60.2 & \\
\hline Female & & 239 & 39.4 & 96 & 40.9 & & 85 & 41.3 & & 58 & 34.9 & \\
\hline Unknown & & 9 & & 0 & & & 1 & & & 8 & & \\
\hline \multicolumn{13}{|l|}{$\mathrm{Age}^{a}$} \\
\hline Mean & 37.94 & & 15.67 & 42.91 & & 16.6 & 34.53 & & 14.36 & 34.18 & & 13.49 \\
\hline \multicolumn{13}{|l|}{ Treatment } \\
\hline New & 333 & 54.9 & & 156 & 66.4 & & 93 & 45.1 & & 84 & 50.6 & \\
\hline Previous & 221 & 36.4 & & 79 & 33.6 & & 113 & 54.9 & & 29 & 17.5 & \\
\hline Unknown & 53 & & & 0 & & & 0 & & & 53 & & \\
\hline \multicolumn{13}{|l|}{ MIRU lineage } \\
\hline Beijing & 411 & 67.7 & & 136 & 57.9 & & 154 & 74.8 & & 121 & 72.9 & \\
\hline Dehli/CAS & 6 & 1 & & 6 & 2.6 & & & & & & & \\
\hline H37Rv-like & 60 & 9.9 & & 31 & 13.2 & & 12 & 5.8 & & 17 & 10.2 & \\
\hline LAM & 36 & 5.9 & & 22 & 9.4 & & 5 & 2.4 & & 9 & 5.4 & \\
\hline NEW-1 & 31 & 5.1 & & 16 & 6.8 & & 10 & 4.9 & & 5 & 3 & \\
\hline URAL & 28 & 4.6 & & 10 & 4.3 & & 11 & 5.3 & & 7 & 4.2 & \\
\hline Haarlem & 26 & 4.3 & & 7 & 3 & & 13 & 6.3 & & 6 & 3.6 & \\
\hline S-type & 3 & 0.5 & & 2 & 0.9 & & & & & 1 & 0.6 & \\
\hline TUR & 1 & 0.2 & & & & & 1 & 0.5 & & & & \\
\hline X-type & 1 & 0.2 & & 1 & 0.4 & & & & & & & \\
\hline Undefined & 4 & 0.7 & & 4 & 1.7 & & & & & & & \\
\hline \multicolumn{13}{|l|}{ MLVA MtbC15-9 } \\
\hline 94-32 (Beijing) & 145 & 23.9 & & 58 & 24.7 & & 49 & 23.8 & & 38 & 22.9 & \\
\hline 100-32 (Beijing) & 70 & 11.5 & & 12 & 5.1 & & 51 & 24.8 & & 7 & 4.2 & \\
\hline \multicolumn{13}{|l|}{ Resistance pattern } \\
\hline Pan-susceptible & 35 & 5.8 & & 21 & 8.9 & & 2 & 1 & & 12 & 7.2 & \\
\hline Other resistances & 279 & 46 & & 127 & 54 & & 66 & 32 & & 86 & 51.8 & \\
\hline MDR-TB & 172 & 28.3 & & 47 & 20 & & 71 & 34.5 & & 54 & 32.5 & \\
\hline Pre XDR-TB & 94 & 15.5 & & 34 & 14.5 & & 50 & 24.3 & & 10 & 6 & \\
\hline XDR-TB & 27 & 4.4 & & 6 & 2.6 & & 17 & 8.3 & & 4 & 2.4 & \\
\hline
\end{tabular}

ankown for Tajikistan and 23 unkown for Kyrgystan 
MIRU-VNTR data was performed using the categorical coefficient. For each 24-loci MIRU-VNTR pattern a unique multiple loci VNTR analysis (MLVA) MtbC15-9 haplotype was assigned by using the MIRU-VNTRplus nomenclature [10]. A cluster was defined as a minimum of two isolates harboring identical genotyping patterns from different patients. Patients with mixed molecular typing patterns were excluded. Drug susceptibility testing was performed by the resazurin microtiter assay [11].

\section{Results}

Twenty four-loci MIRU-VNTR typing and spoligotyping were successfully performed for 607 isolates, while 76 isolates (62 from Uzbekistan, seven from Tajikistan, and seven from Kyrgyzstan) yielded indeterminate results. For 23 isolates ( 9 from Uzbekistan, three from Tajikistan, and 11 from Kyrgyzstan), mixed genotyping patterns were observed. One isolate from Kyrgyzstan had incomplete phenotypic data.
The final study set consisted of 607 isolates of which 235 (38.7\%) originated from Uzbekistan, 206 (33.9\%) from Tajikistan, and 166 (27.3\%) from Kyrgyzstan (Table 1). The male to female ratio of patients was 1.5 and ages ranged from 12 to 85 years with a mean of 37.9 years ( $\mathrm{SD} \pm 15.7$ ). Across the three countries, 333 (54.9\%) were new and 221 (36.4\%) previously treated cases (for 53 patients information regarding previous treatments was missing; Table 1). Thirty five isolates (5.8\%) were pan-susceptible, 293 (48.3\%) MDR-, pre$\mathrm{XDR}$, or XDR-TB, while the remaining 279 isolates (46.0\%) had any other resistance pattern. MDR- and XDR-TB were more prominent among Kyrgyz (33\%) and Tajik (35\%) than among Uzbek isolates (20\%), which was mainly attributable to different re-testing algorithms in the DRS protocols.

Based on the MIRU-VNTR profiles and spoligotyping patterns, 603 isolates were classified into previously

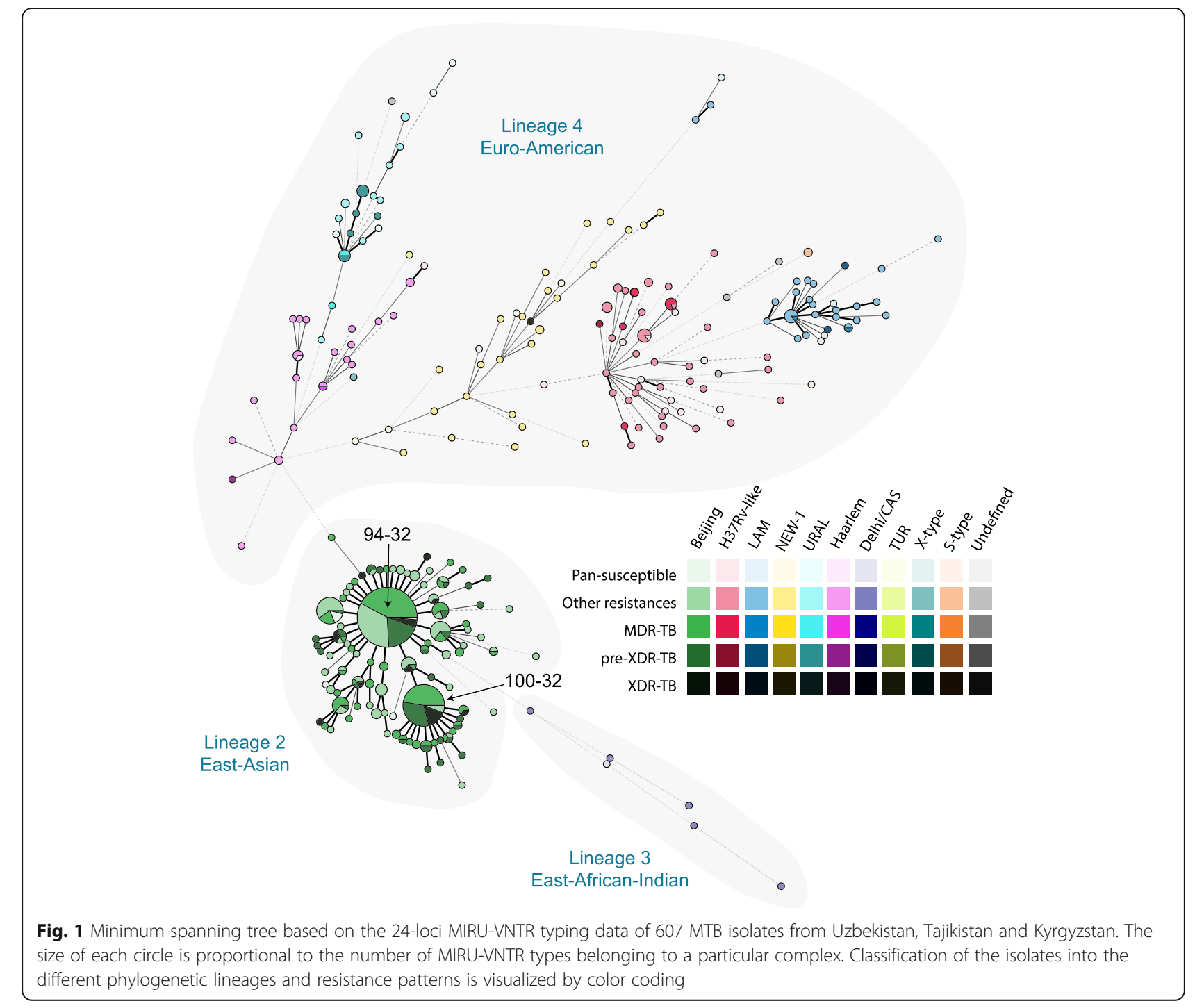


described lineages (Table 1). 411 (67.7\%) isolates belonging to lineage 2 (East-Asian; Beijing genotype) [12]. Six (1.0\%) isolates belonged to lineage 3 (East-African-Indian; Delhi/CAS genotype), and 186 (30.6\%) isolates belonged to lineage 4 (Euro-American) with the predominiating genotypes H37Rv-like (60; 9.9\%), LAM (36; 5.9\%), NEW-1 (31; 5.1\%), URAL (28; 4.6\%), and Haarlem (26; $4.3 \%)$. Four $(0.7 \%)$ isolates could not be linked to a previously described lineage and were classified as "undefined". An MST was calculated (Fig. 1) confirming the UPGMA (unweighted pair group method with arithmetic mean) tree-based genotype classification of MIRU-VNTRplus (data not shown).

Cluster analysis revealed that 382 (62.9\%) of the 607 isolates shared a genotyping pattern with at least one other isolate. They were grouped in 38 clusters ranging from two to 145 isolates. The two largest clusters had haplotypes $94-32(n=145 ; 23.9 \%)$ and $100-32(n=70 ; 11.5 \%)$ (Fig. 1). The prevalence of 94-32 was equally distributed over the CARs whereas cluster 100-32 was particularly prominent in Tajikistan (51 strains; $72.9 \%) .349$ (84.9\%) of the 411 Beijing isolates formed clusters, compared to only $33(16.8 \%)$ of the 196 isolates from other clades.

Beijing strains were more frequently MDR-, pre-XDRor XDR-TB $(265 / 411 ; 64.5 \%)$ than strains of other genotypes (28/196; 14.3\%; no XDR-TB) (Fig. 2). Beijing cluster 94-32 showed the same drug resistance pattern as other Beijing strains, whereas cluster 100-32 was almost completely (66/70; 94.3\%) MDR, pre-XDR, or XDR.

\section{Discussion}

High prevalence of Beijing strains has previously been reported from Central Asia [4]. The star-like shape of the lineage 2/Beijing population in Fig. 1 with two central clones surrounded by layers of single and multi-locus variants is a typical pattern for emerging and expanding MTB populations [13]. The two largest clusters $94-32$ and 10032 were found in all three Central Asian countries and represented more than a third of all study isolates indicating strong population expansion across borders. Our findings confirm the results of a global Beijing population study which reported that 94-32 and 100-32 were predominantly prominent in Uzbekistan, Russia and Eastern European countries and had particularly high clustering rates [13]. The smaller size of 100-32 and its unequal distribution to the three CARs suggest however its more recent arrival in Central Asia and a shorter regional expansion time than of 94-32. This hypothesis is further supported by Mokrousov [14] and Skibe et al. [15] who have already reported 100-32 as minor group $(<4 \%)$ of Beijing isolates in Central Asia while it reached proportions of over $10 \%$ and over $20 \%$ in Eastern Europe and Russia, respectively, and while Skibe et al. observed 94-32 as dominant cluster in Kazakhstan. The extent of cross-border spread and locally restricted transmisison networks can however not be ultimately resolved with MIRU-VNTR cluster data and require further investigation with higher resolution.

High percentages of MDR among Beijing strains has also been reported from other Eastern European countries, like Georgia, Kazakhstan, and Uzbekistan [4, 15, 16]. Higher proportions of MDR- and XDR-TB were also observed within the 100-32 genotype by Merker and colleagues [13]. The authors analyzed the full genomes of MDR-TB strains belonging to MLVA-VNTR haplotypes 94-32 and 100-32 and showed that the maximal divergences were 17 and 23 SNPs, respectively, within genomes of those clusters [13].

Our data indicate that spread of resistant MTB strains, particularly of lineage 2 /Beijing, is an important root of

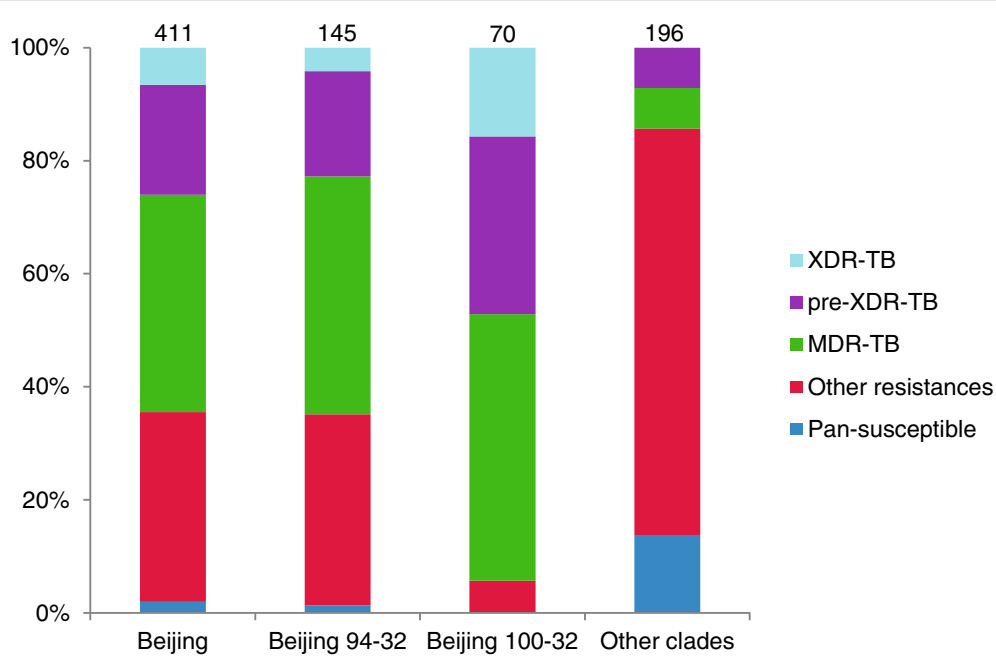

Fig. 2 Proportions of isolates based on drug resistance patterns. The number of isolates with a given genotype/MLVA MtbC15-9 haplotype is given above the corresponding column 
the ongoing MDR-TB epidemic in Central Asia. MIRUVNTR is a powerful tool for investigating population structures and transmission dynamics, however, advances in the field of whole genome sequencing have resulted in molecular methods that can give better resolution and should ideally be used to elucidate specific transmission pathways. Analyzing the full genomes of this or an unbiased and thus even more representative study population could provide an even deeper insight into the MTB population structure in CAR.

\section{Conclusions}

Clusters 94-32 and 100-32 are the dominant genotypes driving population expansion of Beijing strains in the CARs Uzbekistan, Tajikistan, and Kyrgyzstan. The data demonstrate that spread of resistant TB strains, particularly of the Beijing lineage and its clusters $94-32$ and $100-32$, is an important root of the ongoing MDR-TB epidemic in Central Asia. Measures to combat this epidemic need to be improved.

\section{Abbreviations}

CAR: Central Asian Republic; DRS: Drug resistance surveys; MDR: Multi-drugresistant / resistance; MIRU-VNTR: Mycobacterial Interspersed Repetitive Units - Variable Number of Tandem Repeats; MLVA: Multiple loci VNTR analysis; MST: Minimum-spanning tree; MTB: Myocbacterium tuberculosis; TB: Tuberculosis; UPGMA: Unweighted pair group method with arithmetic mean; XDR: Extensively drug resistant / extensive drug resistance

\section{Acknowledgements}

The authors thank Tanja Struve-Sonnenschein, Anja Lüdemann and Tanja Ubben for technical assistance.

\section{Authors' contributions}

$\mathrm{SN}$ and $\mathrm{HH}$ designed the study, AE performed the experiments, $A E$, UA, AK, GK, OK, NP, AR, ES, ZS, and HH collected data, AE, KK, SN and HH analyzed the data, $A E, K K, M M, S N$ and $H H$ wrote the manuscript. All authors read and approved the final manuscript.

\section{Authors' information}

None

\section{Funding}

AE was supported by the International Postdoc Grant from the Swedish Research Council. The funding body had no role in the study design, collection of isolates, analysis, interpretation of data nor in writing the manuscript.

\section{Availability of data and materials}

The datasets used and/or analysed during the current study are available from the corresponding author on reasonable request.

\section{Ethics approval and consent to participate}

Not applicable, but see please see methods section and references 8 and 9 .

\section{Consent for publication}

Not applicable.

\section{Competing interests}

The authors declare that they have no competing interests.

\section{Author details}

${ }^{1}$ Molecular and Experimental Mycobacteriology, Research Center Borstel, Parkallee 1, 23845 Borstel, Germany. ${ }^{2}$ Department of Medical Biochemistry and Microbiology, Uppsala University, IMBIM, Box 582, 75123 Uppsala, Uppsala, Sweden. ${ }^{3}$ Present address: Clinical Genomics, Science for Life
Laboratory, Tomtebodavägen 23 A, 17165 Solna, Sweden. ${ }^{4}$ Present address: School of Engineering Sciences in Chemistry, Biotechnology and Health, Division of Gene Technology, KTH Royal Institute of Technology, Stockholm, Sweden. ${ }^{5}$ Postal address: Science for Life Laboratory, Tomtebodavägen 23 A, 17165 Solna, Sweden. ${ }^{6} \mathrm{WHO}$ Supranational Reference Laboratory of Tuberculosis, IML red GmbH, Institute of Microbiology and Laboratory Medicine, Robert Koch-Allee 2, D-82131 Gauting, Germany. ${ }^{7}$ National Tuberculosis Institute, 90a Ahuunbaen Street, 720075 Bishkek, Kyrgyzstan.

${ }^{8}$ National Reference Laboratory of Tuberculosis, 90a Ahuunbaen Street, 720075 Bishkek, Kyrgyzstan. ${ }^{9}$ Clinical Research Department, London School of Hygiene and Tropical Medicine, London, UK. ${ }^{10}$ National Reference Laboratory of Tuberculosis, Vakhdat District, Dushanbe, Tajikistan. ${ }^{11}$ Republican Specialized Scientific and Practical Medical Center of Tuberculosis and Pulmonology, Alimov Str.1, Tashkent, Uzbekistan100086. ${ }^{12}$ National TB Center National Tuberculosis Program, Dushanbe, Tajikistan. ${ }^{13}$ National Reference Laboratory of Tuberculosis, Alimov Str.1, Tashkent, Uzbekistan100086.

${ }^{14}$ SYNLAB Gauting, SYNLAB Human Genetics Munich, Robert Koch-Allee 2, 82131 Gauting, Germany. ${ }^{15}$ Kuratorium Tuberculosis in the World e.V, Robert Koch-Allee 2, 82131 Gauting, Germany.

Received: 24 February 2019 Accepted: 13 September 2019

Published online: 29 October 2019

\section{References}

1. World Health Organization, Global tuberculosis report 2017. Vol. WHO/HTM/ TB/2017.23. 2017, Geneva, Switzerland.

2. Mokrousov I, et al. Molecular snapshot of mycobacterium tuberculosis population structure and drug-resistance in Kyrgyzstan. Tuberculosis (Edinb). 2013;93(5):501-7.

3. Mokrousov I, et al. Penitentiary population of mycobacterium tuberculosis in Kyrgyzstan: exceptionally high prevalence of the Beijing genotype and its Russia-specific subtype. Infect Genet Evol. 2009;9(6):1400-5.

4. Cox HS, et al. The Beijing genotype and drug resistant tuberculosis in the Aral Sea region of Central Asia. Respir Res. 2005;6:134.

5. Allix-Beguec $\mathrm{C}$, et al. Evaluation and strategy for use of MIRU-VNTRplus, a multifunctional database for online analysis of genotyping data and phylogenetic identification of mycobacterium tuberculosis complex isolates. J Clin Microbiol. 2008;46(8):2692-9.

6. Homolka S, et al. High genetic diversity among mycobacterium tuberculosis complex strains from Sierra Leone. BMC Microbiol. 2008:8:103.

7. Ulmasova DJ, et al. Multidrug-resistant tuberculosis in Uzbekistan: results of a nationwide survey, 2010 to 2011. Euro Surveill. 2013;18(42):pii: 20609.

8. World Health Organization, Guidelines for surveillance of drug resistance in tuberculosis. Vol. WHO/HTM/TB/2009.422. 2009, Geneva, Switzerland.

9. Kamerbeek J, et al. Simultaneous detection and strain differentiation of mycobacterium tuberculosis for diagnosis and epidemiology. J Clin Microbiol. 1997;35(4):907-14.

10. Weniger T, et al. MIRU-VNTRplus: a web tool for polyphasic genotyping of Mycobacterium tuberculosis complex bacteria. Nucleic Acids Res. 2010; 38(Web Server issue):W326-31.

11. Palomino $\mathbf{J C}$, et al. Resazurin microtiter assay plate: simple and inexpensive method for detection of drug resistance in mycobacterium tuberculosis. Antimicrob Agents Chemother. 2002:46(8):2720-2.

12. Gagneux S, Small PM. Global phylogeography of mycobacterium tuberculosis and implications for tuberculosis product development. Lancet Infect Dis. 2007;7(5):328-37.

13. Merker $M$, et al. Evolutionary history and global spread of the mycobacterium tuberculosis Beijing lineage. Nat Genet. 2015;47(3):242-9.

14. Mokrousov I. Insights into the origin, emergence, and current spread of a successful Russian clone of mycobacterium tuberculosis. Clin Microbiol Rev. 2013:26(2):342-60

15. Skiba $Y$, et al. Molecular snapshot of mycobacterium tuberculosis population in Kazakhstan: a country-wide study. Tuberculosis (Edinb). 2015;95(5):538-46.

16. Niemann S, et al. Mycobacterium tuberculosis Beijing lineage favors the spread of multidrug-resistant tuberculosis in the republic of Georgia. J Clin Microbiol. 2010:48(10):3544-50

\section{Publisher's Note}

Springer Nature remains neutral with regard to jurisdictional claims in published maps and institutional affiliations. 